TRANSACTIONS OF THE

AMERICAN MATHEMATICAL SOCIETY

Volume 187, Issue 1,1974

\title{
DUALITY THEORIES FOR METABELIAN LIE ALGEBRAS
}

\author{
BY
}

\author{
MICHAEL A. GAUGER
}

ABSTRACT. This paper is concerned with duality theories for metabelian (2-step nilpotent) Lie algebras. A duality theory associates to each metabelian Lie algebra $N$ with $\operatorname{cod} N^{2}=g$, another such algebra $N_{D}$ satisfying $\left(N_{D}\right)_{D} \simeq$ $N, N_{1} \simeq N_{2}$ if and only if $\left(N_{1}\right)_{D} \simeq\left(N_{2}\right)_{D}$, and if $\operatorname{dim} N=g+p$ then $\operatorname{dim} N_{D}=$ $g+\left(\begin{array}{l}g \\ 2\end{array}\right)-p$. The obvious benefit of such a theory lies in its reduction of the classification problem.

Introduction. The purpose of this paper is to determine the "reasonable" duality theories for metabelian Lie algebras over an algebraically closed field of characteristic zero. If $N$ is a metabelian Lie algebra (i.e. $N^{3}=0$; we are including the abelian Lie algebras as "degenerate" metabelian Lie algebras although this is not standard) such that $\operatorname{cod} N^{2}=g$, a duality theory associates to $N$ another such algebra $N_{D}$, the dual, satisfying the formal properties

1. $\left(N_{D}\right)_{D} \cong N$,

2. $N_{1} \cong N_{2}$ if and only if $\left(N_{1}\right)_{D} \cong\left(N_{2}\right)_{D}$, and

3. if $\operatorname{dim} N=g+p$, then $\operatorname{dim} N_{D}=g+\left(\begin{array}{l}g \\ 2\end{array}\right)-p$.

Two such theories were developed independently by Scheuneman [4] and myself [1]. Their obvious benefit is in the reduction of the classification problem.

In this paper, I define an algebraic duality theory to be an association $N \rightarrow$ $N_{D}$ satisfying conditions a little stronger than the three above, plus a requirement, roughly speaking, that the structure constants of $N_{D}$ depend algebraically on those of $N$. These axioms, which are satisfied by both Scheuneman's and my own duality, result in a uniqueness theorem, hence the identity of the two theories just mentioned. It appeared at first that there were two algebraic duality theories. I am, however, indebted to the referee for detecting an error in my original arguments and for giving a proof of the uniqueness.

A homological approach is taken to the subject by Leger and Luks in [3]. They also arrive at a uniqueness theorem. The main difference between these two papers appears to be their assumption that a duality $D$ is a contravariant functor. Hence, for any homomorphism $\phi: N \rightarrow M$ of metabelian Lie algebras, there is a homomorphism $\phi_{D}: M_{D} \rightarrow N_{D}$. We make no such requirement here.

Due to the generator-relation view given to the classification of metabelian

Received by the editors April 17, 1972.

AMS (MOS) subject classifications (1970). Primary 17B30. 
Lie algebras in [1], the study of algebraic duality theories comes down to studying automorphisms of Grassmann varieties-the projective variety of $p$-dimensional subspaces of a space $V$. Westwick [7] showed that in most cases they are just the obvious ones-induced by $G L(V)$. However, when $2 p=\operatorname{dim} V$, there is an additional type of automorphism arising from a correlation of subspaces; the image of $G L(V)$ is of index two in the automorphism group. This exceptional behavior causes some problems which are resolved in $\$ 3$, a study of the natural representation of $G L(V)$ on $\bigwedge^{p}\left(\bigwedge^{2} V\right)$. The results generated concerning automorphisms of Grassmann varieties are summarized in the First Main Theorem. I would like to thank my colleague John Fogarty for some highly beneficial discussions on algebro-geometric aspects of the problem, and for providing the proof of a key result (Proposition 3) which enabled me to study automorphisms of Grassmann varieties in an affine situation, and thus employ the elegant representation theory of simple Lie algebras.

1. Metabelian Lie algebras and duality theories. In the following, all algebras are finite-dimensional over an algebraically closed field $K$ of characteristic zero. A Lie algebra $N$ is said to be metabelian if $N^{3}=0$ where $N^{n+1}=\left[N^{n}, N\right]$. Let $V$ be a $g$-dimensional $K$-vector space having a basis $x_{1}, \cdots, x_{g}$ which is fixed throughout this paper. We define $N(2, g)$ to be the vector space $V \oplus \wedge^{2} V$ made into a metabelian Lie algebra by bilinearly extending the rules.

\section{In [1] I established}

$$
\begin{gathered}
{\left[x_{i}, x_{j}\right]=x_{i} \wedge x_{j}, \quad\left[x_{i} \wedge x_{j}, x_{k}\right]=0=\left[x_{i}, x_{j} \wedge x_{k}\right],} \\
{\left[x_{i} \wedge x_{j}, x_{m} \wedge x_{n}\right]=0 .}
\end{gathered}
$$

Theorem 1. If $N$ is a metabelian Lie algebra and $\operatorname{cod} N^{2}=g, N$ is isomorphic to $N(2, g) / I$ for some ideal $I$ contained in $\Lambda^{2} V=N(2, g)^{2}$. Every subspace $S$ of $\bigwedge^{2} V$ is an ideal, and if $N=N(2, g) / S$ then $N$ is a metabelian Lie algebra and $\operatorname{cod} N^{2}=g$. Furthermore, if $I$ and $J$ are subspaces of $\wedge^{2} V$, then $N(2, g) / I \cong$ $N(2, g) / J$ if and only if there is a $\theta \in G L(V)$ such that $\Lambda^{2}(\theta)(I)=J$.

Convention. Denote by $\wedge^{r}$ the homomorphism from $G L(V)$ into $G L\left(\Lambda^{r} V\right)$ given by $\wedge^{r}(\theta)\left(v_{1} \wedge \ldots \wedge v_{r}\right)=\theta\left(v_{1}\right) \wedge \ldots \wedge \theta\left(v_{r}\right)$ on decomposable r-vectors.

The duality theory was established in the following way. There is a canonical nondegenerate pairing $\left(\right.$, ) between $\wedge^{2} V$ and $\wedge^{2}\left(V^{*}\right)$ given on decomposable 2-vectors by

$$
(v \wedge w, \alpha \wedge \beta)=\alpha(v) \beta(w)-\beta(v) \alpha(w)
$$

where $v \wedge w \in \wedge^{2} V$ and $a \wedge \beta \in \wedge^{2}\left(V^{*}\right)$. Let $\rho$ be the representation $\wedge^{2}$ of $G L(V)$ on $\wedge^{2} V$ and let $\rho^{*}$ be its contragredient with respect to the pairing of (2). Let $G_{p}(W)$ represent the projective Grassmann variety of $p$-dimensional sub- 
spaces of the finite-dimensional space $W$. Set $n=\left(\begin{array}{l}8 \\ 2\end{array}\right)$ and consider the map $S \rightarrow$ $S^{0}$ (right orthogonal complementation with respect to (2)) from $G_{p}\left(\bigwedge^{2} V\right)$ onto $G_{n-p}\left(\bigwedge^{2}\left(V^{*}\right)\right)$. It is an orbit preserving bijection when $G L(V)$ acts on $\wedge^{2} V$ and $G L\left(V^{*}\right)$ acts on $\wedge^{2}\left(V^{*}\right)$ since

$$
(\rho(\theta)(S))^{0}=\rho^{*}(\theta)\left(S^{0}\right) \text { and } \rho^{*}(\theta)=\bigwedge^{2}\left({ }^{t} \theta^{-1}\right)
$$

where $S \in G_{p}\left(\wedge^{2} V\right)$ and " $t$ " denotes transpose. Make the vector space $V^{*} \oplus$ $\bigwedge^{2}\left(V^{*}\right)$ into a metabelian Lie algebra isomorphic to $N(2, g)$ by substituting the corresponding elements of the dual basis $x_{1}^{*}, \ldots, x_{g}^{*}$ into the relations (1).

Definition. Let $N=N(2, g) / S=V \oplus \wedge^{2} V / S$ where $S$ is a subspace (ideal) of $\wedge^{2} V$. The algebra $N^{0}=V^{*}=\oplus \wedge^{2}\left(V^{*}\right) / S^{0}$ will be called the dual of $N$.

Theorem 2. Let $N, N_{i}$ be metabelian Lie algebras having derived algebras of codimension $g$. Then

(i) $\left(N^{0}\right)^{0} \cong N$,

(ii) $N_{1} \cong N_{2}$ if and only if $N_{1}^{0} \cong N_{2}^{0}$,

(iii) if $\operatorname{dim} N=g+p$ then $\operatorname{dim} N^{0}=g+\left(\begin{array}{l}g \\ 2\end{array}\right)-p$, and $\operatorname{cod}\left(N^{0}\right)^{2}=g$,

(iv) if $N_{i}=N(2, g) / S_{i}$ and $\theta \in G L(V)$ induces an isomorphism of $N_{1}$ onto $N_{2}$ (i.e. $\left.\bigwedge^{2}(\theta)\left(S_{1}\right)=S_{2}\right)$, then ${ }^{t} \theta^{-1} \in G L\left(V^{*}\right)$ induces an isomorphism from $N_{1}^{0}$ onto $N_{2}^{0}$.

Remark. If an algebra $N$ is viewed as $V^{*} \oplus \bigwedge^{2}\left(V^{*}\right) / T$ for some subspace $T$ of $\wedge^{2}\left(V^{*}\right)$ then its dual is given by $V \oplus \wedge^{2} V /{ }^{0} T$ where ${ }^{0}()$ denotes left orthogonal complementation with respect to (2). Thus, up to isomorphism, the notion of dual is independent of the space $V$.

A duality whose formal properties are identical to those of the duality established here was constructed earlier by Scheuneman [4] using the cohomology ring of the Lie algebra. We will show later, as a consequence of the uniqueness theorem for algebraic dualities (Second Main Theorem), the identity of Scheuneman's theory with the one given above.

Motivated by Theorem 2 and the fact that ()$^{0}: G_{p}\left(\bigwedge^{2} V\right) \rightarrow G_{n-p}\left(\bigwedge^{2}\left(V^{*}\right)\right)$ is an algebraic isomorphism (i.e. an isomorphism of projective varieties), we make the following

Definition. An algebraic duality theory for metabelian Lie algebras consists of a mapping $N \rightarrow N_{D}$ for each $g \in \mathbf{Z}_{\text {pos }}$ of the set of metabelian Lie algebras $N$ with $\operatorname{cod} N^{2}=g$ onto itself, given by

$$
\begin{aligned}
& N=V \oplus \wedge^{2} V / S \rightarrow N_{D}=V^{*} \oplus \bigwedge^{2}\left(V^{*}\right) / D(S), \\
& M=V^{*} \oplus \wedge^{2}\left(V^{*}\right) / T \rightarrow M_{D}=V \oplus \wedge^{2} V / D(T),
\end{aligned}
$$


where $V$ is a fixed $g$-dimensional $K$-vector space and $V^{* *}\left(G L\left(V^{* *}\right)\right)$ is identified with $V(G L(V))$ in the canonical way, and where

(I) if $V \oplus \wedge^{2} V / S \cong V^{*} \oplus \bigwedge^{2}\left(V^{*}\right) / T$ then $V^{*} \oplus \wedge^{2}\left(V^{*}\right) / D(S) \cong V \oplus$ $\bigwedge^{2} V / D(T)$

(II) $D: G_{p}\left(\bigwedge^{2} V\right) \rightarrow G_{n-p}\left(\bigwedge^{2}\left(V^{*}\right)\right), D: G_{p}\left(\bigwedge^{2}\left(V^{*}\right)\right) \rightarrow G_{n-p}\left(\bigwedge^{2} V\right)$ are algebraic isomorphisms and $n=\left(\begin{array}{l}g \\ 2\end{array}\right)$,

(III) $\left(N_{D}\right)_{D} \cong N$, and

(IV) if $\theta \in G L(V)\left(G L\left(V^{*}\right)\right)$, then $D \circ \wedge^{2}(\theta)(S)=\bigwedge^{2}\left({ }^{t} \theta^{-1}\right) \circ D(S)$ for all subspaces $S$ of $\bigwedge^{2} V\left(\bigwedge^{2}\left(V^{*}\right)\right)$.

Two such theories $D_{1}, D_{2}$ are said to be equal if $N_{D_{1}} \cong N_{D_{2}}$ for all $N$.

Notice that (I) says the duality is well defined up to isomorphism, that is, it is independent of the underlying space of generators $V$. Also, (IV) implies that if $\theta \in G L(V)$ induces an isomorphism of $N_{1}=V \oplus \wedge^{2} V / S$ onto $N_{2}=V \oplus \wedge^{2} V / T$, then ${ }^{t} \theta^{-1} \in G L\left(V^{*}\right)$ induces an isomorphism of $\left(N_{1}\right)_{D}$ onto $\left(N_{2}\right)_{D}$. Hence, in conjunction with (III), we get the weaker condition

(V) $N_{1} \cong N_{2}$ if and only if $\left(N_{1}\right)_{D} \cong\left(N_{2}\right)_{D}$.

The theory established before using orthogonal complementation with respect to (2) is seen to satisfy all these axioms.

Now suppose $D_{1}, D_{2}$ are algebraic duality theories and let $V$ be as in the def inition. Consider $D_{1}, D_{2}: G_{p}\left(\bigwedge^{2} V\right) \rightarrow G_{n-p}\left(\bigwedge^{2}\left(V^{*}\right)\right)$. By (II), $D_{2}^{-1} \circ D_{1}$ is an automorphism of $G_{p}\left(\bigwedge^{2} V\right)$, say $\rho$. Now $D_{2} \circ \rho=D_{1}$ and by (IV), if $\theta \in G L(V)$ then $D_{2} \circ \rho \circ \bigwedge^{2}(\theta)=D_{1} \circ \bigwedge^{2}(\theta)=\bigwedge^{2}\left({ }^{t} \theta^{-1}\right) \circ D_{1}=\bigwedge^{2}\left({ }^{t} \theta^{-1}\right) \circ D_{2} \circ \rho=D_{2} \circ$ $\bigwedge^{2}(\theta) \circ \rho$. Thus $\rho \circ \bigwedge^{2}(\theta)=\bigwedge^{2}(\theta) \circ \rho$ for all $\theta \in G L(V)$, that is, $\rho$ centralizes the image of $G L(V)$ in Aut $G_{p}\left(\wedge^{2} V\right)$-the group of automorphisms of the variety $G_{p}\left(\bigwedge^{2} V\right)$. A careful study of this group (Westwick [7]) and of the representation $\wedge^{p} p \circ \wedge^{2}$ of $G L(V)$ is enough to show $\rho=I d$ except in one special case. This exceptional case yields no new duality theories however, and a uniqueness theorem follows.

As was pointed out to me by the referee, axiom (III) apparently plays no role in the preceding discussion and what follows later. It is not apparent however in what way it comes from (I), (II), and (IV). I have thus included it is the list of axioms only to emphasize that the following situation cannot occur, namely that two nonisomorphic algebras might have isomorphic duals.

2. Automorphisms of $G_{p}(W)$. Let $W$ be any $k$-vector space and let $G_{p}(W)$ be the projective Grassmann variety of $p$-dimensional subspaces of $W$. If $D_{p}(W)=$ $\left\{w_{.1} \wedge \ldots \wedge w_{p} \mid w_{i} \in W\right\}$ is the homogeneous affine subvariety of $\wedge^{p} W$ consisting of decomposable $p$-vectors, then $G_{p}(W)$ is the projectivization of $D_{p}(W)$. The 
next three results give a complete picture of the structure of Aut $G_{p}(W)$.

Proposition 3. Every automorphism of $G_{p}(W)$ is induced by a linear transformation of $\wedge^{p} W$ stabilizing $D_{p}(W)$.

Proof (J. Fogarty). Let $\mathcal{X}=G_{p}(W)$ and let $\sigma \in$ Aut $(\mathcal{X})$. $\sigma$ induces an automorphism of Pic $(\mathscr{X})=H^{1}\left(\mathscr{X}, \Theta_{\mathscr{X}}{ }^{*}\right)$. The latter group, however, is $\mathrm{Z}$. Since $\sigma$ takes positive line bundles to positive line bundles, it must induce the identity on Pic $(X)$. If $L$ is a line bundle in the class $l \in \operatorname{Pic}(\mathscr{X})$, the Plucker embedding of $\mathcal{X}$ is given by sections of $L$. Thus $\sigma$ is induced by an element of PGL $\left(H^{0}(X, L)\right)$.

With this result, to study Aut $G_{p}(W)$ it is necessary to find the stabilizer in $G L\left(\bigwedge^{p} W\right)$ of $D_{p}(W)$, written Stab $D_{p}(W)$, which was accomplished by Westwick [7]. His main result is the

Theorem 4. Let $W$ be an n-dimensional vector space over an algebraically closed field and let $T$ be a linear transformation of $\wedge^{r} W$ stabilizing $D_{r}(W)$. Then $T$ is a compound (i.e. $T=\wedge^{r} \theta$ for some $\theta \in G L(W)$ ) except possibly when $n=2 r$ in which case $T$ could also be the product of a compound with a linear transformation $C$ induced by a correlation of r-dimensional subspaces of $W$. In the latter case, $\left(\right.$ Stab $\left.D_{r}(W): \bigwedge^{r} G L(W)\right)=2$.

Proof [7,pp. 1126-1127]. Since in the case $n=2 r, A^{r} G L(V)$ is of index 2 in the stabilizer, the correlation $C$ can be taken to be the same for all $T$ which are not compounds. For out purposes it is advantageous to choose $C$ as follows. Let $W=\wedge^{2} V, V=\left\langle x_{1}, \cdots, x_{g}\right\rangle$ and suppose $2 r=\operatorname{dim} \wedge^{2} V$. Set $x_{i j}=x_{i} \wedge x_{j}$ $(i<j)$ and order the pairs $(i, j), 1 \leq i j<j \leq g$ lexicographically. Then

$$
\left\{x_{i_{1} j_{1}} \wedge \ldots \wedge x_{i_{r} j_{r}} \mid\left(i_{1}, j_{1}\right)<\left(i_{2}, j_{2}\right)<\ldots<\left(i_{r}, j_{r}\right)\right\}
$$

is an ordered basis of $\wedge^{r} w$. Let $C$ be the linear extension of the map given by $C\left(x_{i_{1} j_{1}} \wedge \ldots \wedge x_{i_{r} j_{r}}\right)=x_{k_{1} l_{1}} \wedge \ldots \wedge x_{k_{r} l_{r}}$ where

$$
\left(k_{1}, l_{1}\right)<\left(k_{2}, l_{2}\right)<\ldots<\left(k_{r}, l_{r}\right)
$$

and where $\left\{\left(i_{t}, j_{t}\right),\left(k_{t}, l_{t}\right)\right\}_{t=1}^{r}=\{(i, j) \mid 1 \leq i<j \leq g\}$.

Thus, if $T \in \operatorname{Stab} D_{r}(W)$ and $T \notin \wedge^{r} G L(W)$ then

$$
T=C \circ \wedge^{r}(\theta)
$$

where $\theta \in G L\left(\bigwedge^{2} V\right)$ and $C$ is given by (4).

Lemma 5. The kernel of the projection homomorpbism $\pi$ : Stab $D_{p}\left(\bigwedge^{2} V\right) \rightarrow$ Aut $G_{p}\left(\bigwedge^{2} V\right)$ consists entirely of scalars.

Proof. Suppose $2 p \neq \operatorname{dim} \wedge^{2} V$, then by Theorem 4 the stabilizer consists of compounds. Suppose $\pi\left(\bigwedge^{p}(\theta)\right)=I d$, that is, $\theta$ stabilizes every $p$-dimensional 
subspace of $\Lambda^{2} V$. Let $v$ be any vector in $\Lambda^{2} V$ and let $s_{1}, \cdots, s_{m}$ be a collection of $p$-dimensional subspaces of $\Lambda^{2} V$ with $\langle v\rangle=\bigcap_{i=1}^{m} s_{i}$. Then $\theta(\langle v\rangle)=\langle v\rangle$ since $\theta$ stabilizes each $S_{i}$, that is, every vector in $\Lambda^{2} V$ is a $\theta$-eigenvector. Hence $\theta$ is a scalar, and so is $\bigwedge^{p}$.

Suppose $2 p=\operatorname{dim} \wedge^{2} V$ and suppose $\pi(\rho)=$ Id where $\rho \in \operatorname{Stab} D_{p}\left(\bigwedge^{2} V\right)$. If $\rho$ is a compound proceed as before to show $\rho$ is a scalar. If $\rho$ is not a compound it is of the type $\Lambda^{p}(\theta) \circ C$ where $C$ is given by (4). Let $w_{1}, \ldots, w_{2 p}$ be the ordered basis $\left\{x_{i j} \mid i<j\right\}$ of $\wedge^{2} V$ where $x_{i j}$ is as before. Then $\theta\left(w_{p+1}\right) \wedge \cdots \wedge \theta\left(w_{2 p}\right)=$ $\wedge^{p}(\theta) \circ C\left(w_{1} \wedge \ldots \wedge w_{p}\right) \epsilon\left\langle w_{1} \wedge \ldots \wedge w_{p}\right\rangle$ since $\pi(\rho)=$ Id. Hence $\theta\left(w_{p+1}\right) \epsilon$ $\left\langle w_{1}, \cdots, w_{p}\right\rangle$. Similarly, considering $\wedge^{p}(\theta) \circ C\left(w_{1} \wedge w_{p+2} \wedge w_{p+3} \wedge \ldots \wedge w_{2 p}\right)$ and $\wedge p(\theta) \circ C\left(w_{2} \wedge w_{p+2} \wedge w_{p+3} \wedge \ldots \wedge w_{2 p}\right)$ we see that $\theta\left(w_{p+1}\right) \in\left\langle w_{1}, \ldots, w_{p}\right\rangle \cap$ $\left\langle w_{1}, w_{p+2}, w_{p+3}, \cdots, w_{2 p}\right\rangle \cap\left\langle w_{2}, w_{p+2}, w_{p+3}, \cdots, w_{2 p}\right\rangle=0$, which is a contradiction.

For the following discussion let $G_{1}=\bigwedge^{2}(G L(V)) \subset G L\left(\wedge^{2} V\right), G_{2}=\wedge^{p} G_{1} \subset$ $G L\left(\wedge^{p} \wedge^{2} V\right), G_{3}=\pi\left(G_{2}\right) \subset$ Aut $G_{p}\left(\wedge^{2} V\right)$ where $\pi$ is given in Lemma 5. Also, let $\rho \in$ Aut $G_{p}\left(\wedge^{2} V\right)$ and suppose $\rho$ centralizes $G_{3}$. By Proposition 3, there is an $\omega \in G L\left(\wedge^{p} \wedge^{2} V\right)$ such that

$$
\pi(\omega)=\rho
$$

Lemma 6. Let $\rho, \omega, G_{i}$ be as above. Then $\omega$ centralizes $G_{2}$.

Proof. $\rho$ centralizes $G_{3}$, so by Lemma 5 and equation (6), for each $\theta \in G_{2}$ we have $\omega^{-1} \theta \omega=a_{\theta} \cdot \theta$ where $\alpha_{\theta} \in K$. Taking determinants we see that $\alpha_{\theta}$ is an lth root of unity where $l=\operatorname{dim} \wedge^{p} \wedge^{2} V$. Thus, set $G_{2}^{\prime} \doteq\left\{g \in G_{2} \mid \omega^{-1} g \omega=g\right\}$. By the above, $G_{2}^{\prime}$ is a subgroup of index at most $l$ in $G_{2}$, and quite obviously the defining condition of $G_{2}^{\prime}$ makes it Zariski-closed in $G_{2}$. Furthermore, $G_{2}$ is Zariskiirreducible (being the image of $G L(V)$ by $\wedge^{p} \circ \wedge^{2}$ ), hence $G_{2}^{\prime}=G_{2}$.

Lemma 7. Let $\rho, \omega, G_{i}$ be as above and suppose $2 p \neq \operatorname{dim} \wedge^{2} V$. Then $\rho$ is the identity element.

Proof. By Theorem 4, $\omega=\bigwedge^{p}(\theta)$ where $\theta \in G L\left(\bigwedge^{2} V\right)$. Proceeding just as in the proof of Lemma 6 (since the kernel of $\wedge^{p}: G L(W) \rightarrow G L\left(\wedge^{p} W\right)$ consists of $p$ th roots of unity) one can see that $\theta$ centralizes $G_{1}=\bigwedge^{2} G L(V)$. But, $G L(V)$ acts irreducibly on $\Lambda^{2} V$. If $s l(V)$ is the Lie algebra of trace-zero linear transformations on $V$, the differential of $\wedge^{2}$ restricted to $s l(V)$ is the second fundamental (irreducible) representation of $s l(V)$ (see [2, pp. 225-227]). Hence, by Burnside's theorem, $\theta$ is a scalar and $\rho=\pi\left(\wedge^{p}(\theta)\right)=\mathrm{Id}$.

Remark. The corresponding result when $\operatorname{dim} V=2 p$ is complicated by the exceptional structure of Aut $G_{p}\left(\bigwedge^{2} V\right)$. It requires a careful look at the representation $\wedge^{p} \circ \wedge^{2}$ of $G L(V)$ on $\wedge^{p} \wedge^{2} V$. Were this representation irreducible the proof 
would proceed quite easily. In general it is not. However, it proves quite useful that $\wedge^{p} \wedge^{2} V$ has lots of simple submodules of multiplicity one (i.e. simple submodules which, up to isomorphism, appear only once in a decomposition of $\wedge^{p} \wedge^{2} V$ as a direct sum of simple submodules).

3. The $G L(V)$-module $\wedge^{p} \wedge^{2} V$. In this section we are concerned with determining some of the simple $G L(V)$-submodules of $\wedge^{p} \wedge^{2} V$ of multiplicity one when $2 p=\operatorname{dim} \wedge^{2} V$ (this can occur if and only if $\operatorname{dim} V \equiv 0,1(4)$ ). Since $\wedge^{p} \circ \bigwedge^{2}$ takes scalars to scalars, the $G L(V)$ and $S L(V)$ submodules are identical. Also, for any $r, d \bigwedge^{r}$ (the differential of $\bigwedge^{r}$ ) is the representation of $s l(V)$ given by

$$
d \wedge^{r}(l)\left(v_{1} \wedge \ldots \wedge v_{r}\right)=\sum_{i=1} v_{1} \wedge \ldots \wedge v_{i-1} \wedge l\left(v_{i}\right) \wedge v_{i+1} \wedge \ldots \wedge v_{r}
$$

Making $\wedge^{p} \wedge^{2} V$ an $s l(V)$-module according to the representation $d\left(\wedge^{p} \circ \bigwedge^{2}\right)=$ $d \Lambda^{p} \circ d \Lambda^{2}$, the $G L(V)$ and $s l(V)$-submodules are identical. Using the theory of e-extreme modules and highest weights [2, Chapter 7] we can now begin to pick out some simple $s l(V)(G L(V))$-submodules of multiplicity one.

Let $l+1=\operatorname{dim} V=g$ and let $V=\left\langle x_{1}, \cdots, x_{g}\right\rangle$ as before. Let $e_{i j}$ be the element of $s l(V)$ whose matrix with respect to $\left\{x_{i}\right\}_{i=1}^{g}$ has a " 1 " in the $(i, j)$-position and zeros elsewhere. Set $e_{i}=e_{i, i+1}$ for $i=1, \cdots, l$, set $b_{i}=e_{i i}-e_{i+1, i+1}$ and let $H$ be the Cartan subalgebra of $s l(V)$ spanned by the $b_{i}$ 's. Define $\Lambda_{i} \in H^{*}$ by

$$
\begin{aligned}
& \Lambda_{1}\left(b_{1}\right)=1, \quad \Lambda_{1}\left(b_{j}\right)=0 \text { for } j>1, \\
& \Lambda_{i}\left(b_{i-1}\right)=-1, \quad \Lambda_{i}\left(b_{i}\right)=1, \quad \Lambda_{i}\left(b_{j}\right)=0 \text { otherwise, } \\
& \Lambda_{l+1}\left(b_{l}\right)=-1, \quad \Lambda_{l+1}\left(b_{j}\right)=0 \quad \text { for } j \neq l \text {, }
\end{aligned}
$$

where $i=2, \cdots, l$.

Then $\left\langle x_{i}\right\rangle$ is a weight space for $H$ of weight $\Lambda_{i} ; \Lambda_{1}, \cdots, \Lambda_{l}$ are linearly independent and $\Sigma_{i=1}^{l+1} \Lambda_{i}=0$. As before set $x_{i j}=x_{i} \wedge x_{j}(i<j)$ and order the pairs $(i, j), 1 \leq i<j \leq g=l+1$ lexicographically. Then $\left\{x_{i j}\right\}$ is a basis of $\wedge^{2} V$ and notice that if $b \in H$ then

$$
d \wedge^{2}(b)\left(x_{i j}\right)=b\left(x_{i}\right) \wedge x_{j}+x_{i} \wedge b\left(x_{j}\right)=\left(\Lambda_{i}+\Lambda_{j}\right)(b) x_{i} \wedge x_{j} \cdot
$$

Similarly, the set $\left\{x_{i_{1} j_{1}} \wedge \ldots \wedge x_{i_{p} j_{p}} \mid\left(i_{1}, j_{1}\right)<\left(i_{2}, j_{2}\right)<\ldots\right\}$ is a basis of $\wedge^{p} \wedge^{2} V$ and its members will be called standard decomposable vectors. Notice that $\left\langle x_{i_{1} j_{1}} \wedge \ldots \wedge x_{i_{p} j_{p}}\right\rangle$ is a weight space for $H$ of weight $\sum_{k=1}^{p}\left(\Lambda_{i_{k}}+\Lambda_{j_{k}}\right)$.

Definition. An $s l(V)$-module $M$ is called $e$-extreme if it is cyclic and a generator $v \in M$ can be chosen so that $v$ is a weight vector for $H$ and $e_{i}(v)=0$ for $i=1, \ldots, l$. Such a vector $v$ is called a primitive vector and its weight is called the highest weight of $M\left[2\right.$, Chapter 7]. Also if $y \in M, \lambda \in H^{*}$ and $b(y)=\lambda(b) y$ for all $b \in H$, then we will write $w t(y)=\lambda$. 
Lemma 8. A finite-dimensional e-extreme module is simple.

Proof. See the discussion on p. 229 of [2].

Now consider $\wedge^{p} \Lambda^{2} V$ as an $s l(V)$-module. It is a direct sum of simple submodules, and, furthermore, any simple $s l(V)$-module is e-extreme [2, Chapter 7]. Thus, to find simple submodules one looks for primitive vectors. To find the multiplicities of these submodules we compare the weights of the primitive vectors, since two e-extreme submodules are isomorphic if and only if they have the same highest weight.

Definition. Let $x=x_{i_{1} j_{1}} \wedge \ldots \wedge x_{i_{p} j_{p}}$ be a standard decomposable vector. For $k=1, \cdots, l+1$ we define the $k$-index of $x$, written $I_{k}(x)$, to be the number of times the index $k$ appears on the list $\left\{i_{1}, j_{1}, i_{2}, j_{2}, \ldots\right\}$.

Lemma 9. Let $x=x_{i_{1} j_{1}} \wedge \ldots \wedge x_{i_{p} j_{p}}$ and $y=x_{k_{1} l_{1}} \wedge \ldots \wedge x_{k_{p} l_{p}}$ be 2 standard decomposable vectors. Then $w t(x)=w t(y)$ if and only if $I_{k}(x)=I_{k}(y)$ for $k=$ $1, \cdots, l+1$.

Proof. Recall that $w t(x)=\Sigma_{s=1}^{p}\left(\Lambda_{i_{s}}+\Lambda_{j_{s}}\right)$. Thus $(\Leftarrow)$ is direct. $(\Rightarrow)$ Let $s=I_{l+1}(x), t=I_{l+1}(y)$. Since $\Lambda_{l+1}=-\left(\Lambda_{1}+\cdots+\Lambda_{l}\right)$ we have

$$
\begin{aligned}
& w t(x)=\left(I_{1}(x)-s\right) \Lambda_{1}+\cdots+\left(I_{l}(x)-s\right) \Lambda_{l}, \\
& w t(y)=\left(I_{1}(y)-t\right) \Lambda_{1}+\cdots+\left(I_{l}(y)-t\right) \Lambda_{l} .
\end{aligned}
$$

Equating coefficients, since $\Lambda_{1}, \cdots, \Lambda_{l}$ are linearly independent, we arrive at

$$
I_{k}(x)-s=I_{k}(y)-t, \quad k=1, \ldots, l .
$$

Suppose $t>s$. Then $I_{k}(y)=I_{k}(x)+(t-s)$ and

$$
\begin{aligned}
2 p & =\sum_{k=1}^{l+1} I_{k}(y)=t+\sum_{k=1}^{l} I_{k}(y)=t+\sum_{k=1}^{l}\left(I_{k}(x)+(t-s)\right) \\
& =t+l(t-s)+\sum_{k=1}^{l} I_{k}(x)>\sum_{k=1}^{l+1} I_{k}(x)=2 p
\end{aligned}
$$

which is clearly impossible. Reversing the roles of $x$ and $y$ we see $s=t$ and the result follows from (9).

Theorem 10. Let $\mathcal{E}$ be the collection of all standard decomposable vectors of the type

$$
\begin{aligned}
y_{m}= & x_{12} \wedge x_{13} \wedge \ldots \wedge x_{1 m_{1}} \wedge x_{23} \wedge x_{24} \wedge \ldots \wedge x_{2 m} \\
& \wedge \cdots \wedge x_{q q+1} \wedge x_{q q+2} \wedge \ldots \wedge x_{q m_{q}}
\end{aligned}
$$

where $m_{1} \geq m_{2} \geq \cdots \geq m_{q}$ and $\sum_{i=1}^{q}\left(m_{i}-i\right)=p$. Then 
(i) the elements of $\mathcal{E}$ are primitive with pairwise distinct weights, and

(ii) there is no other primitive vector in $\bigwedge^{p} \bigwedge^{2} V$ with a weight equal to that of one in $\xi_{\text {. }}$

Proof. Notice that the effect of $e_{i}$ on $x_{j}$ is either to kill it or lower its index by one. This remark together with (7) shows that $e_{i}$ kills the elements of $\xi_{\text {. The }}$ rest of (i) and (ii) follows from

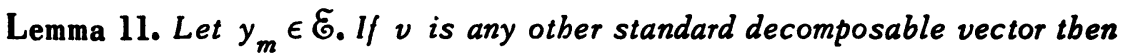
$w t(v) \neq w t\left(y_{m}\right)$.

Proof. By Lemma 9, $w t(v)=w t\left(y_{m}\right)$ implies $I_{k}(v)=I_{k}\left(y_{m}\right)$ for $k=1, \cdots, l+1$. Thus $v$ must have $m_{1}-1$ factors of the type $x_{1 *}$. But $I_{m_{1+1}}\left(y_{m}\right)=0$, thus the *'s must be filled in by the indices $2,3, \ldots, m_{1}$. Thus $v=x_{12} \wedge \ldots \wedge x_{1 m_{1}} \wedge$ .... Proceed by induction to complete the proof.

Definition. If $x \in \wedge^{p} \wedge^{2} V$ let $\langle x\rangle_{\text {mod }}$ denote the $G L(V)(s l(V))$-submodule generated by $x$.

Theorem 12. The simple submodules of $\bigwedge^{p} \Lambda^{2} V$ of the type $\left\langle y_{m}\right\rangle_{\bmod }$ where $y_{m} \in \mathcal{E}$ are of multiplicity one.

Proof. $\wedge^{p} \wedge^{2} V$ is a direct sum of simple submodules each of which is $e$-extreme. Furthermore, simple modules are isomorphic if and only if their highest weights agree. The result now follows from Theorem 10 .

4. Automorphisms of $G_{p}\left(\bigwedge^{2} V\right)$ when $2 p=\operatorname{dim} \bigwedge^{2} V$ and the uniqueness theorem. We return now to the situation of $\$ 2$. Let $G_{1}=\bigwedge^{2} G L(V) \subset G L\left(\wedge^{2} V\right), G_{2}=$ $\wedge^{p} G_{1} \subset G L\left(\wedge^{p} \wedge^{2} V\right)$, and $G_{3}=\pi\left(G_{2}\right)$ where, $\pi$ is given in Lemma 5. Suppose $\rho \in$ Aut $G_{p}\left(\wedge^{2} V\right)$ and it centralizes $G_{3}$. By Proposition 3 there is an $\omega \epsilon$ $G L\left(\wedge^{p} \wedge^{p} V\right)$ such that

$$
\pi(\omega)=\rho \text {. }
$$

In this section we will also assume that $\operatorname{dim} \bigwedge^{2} V=2 p ;$ thus $\operatorname{dim} V \equiv 0,1$ (4).

Lemma 13. Suppose $2 p=\operatorname{dim} \wedge^{2} V$ and $\operatorname{dim} V \geq 5$, and let $\rho, \omega, G_{i}$ be as above. Then $\rho=\mathrm{Id}$.

Proof. As in Lemma 6 we see that $\omega^{\circ}$ centralizes $G_{2}$. By Theorem 4 either $\omega=C \circ \bigwedge^{p}(\theta)$ where $\theta \in G L\left(\bigwedge^{2} V\right)$ and $C$ is given by (4), or else $\omega=\bigwedge^{p}(\theta)$. In the latter case proceed just as in the proof of Lemma 7 to show $\rho=$ Id. So assume now that $\omega=C \circ \wedge^{p}(\theta)$ and we will see what goes wrong.

Recall that $C\left(x_{i_{1} j_{1}} \wedge \ldots \wedge x_{i_{p} j_{p}}\right)=x_{k_{1} l_{1}} \wedge \ldots \wedge x_{k_{p} l_{p}}$ where $\left(k_{1}, l_{1}\right)<$ $\left(k_{2}, l_{2}\right)<\ldots$ and $\left\{\left(i_{s}, i_{s}\right),\left(k_{s}, l_{s}\right)\right\}_{s=1}^{p}=\{(i, j) \mid 1 \leq i<j \leq g\}$. Thus $C^{2}=\mathrm{Id}$, and hence 


$$
C \circ \omega=\bigwedge^{p}(\theta) \text {. }
$$

Let $\mathcal{E}$ be as in Theorem 10. Now by Lemma $6, \omega$ centralizes $G_{2}$, so $\omega$ is an $s l(V)(G L(V))$-isomorphism of $\bigwedge^{p} \bigwedge^{2} V$. Since the simple submodules generated by elements of $\mathcal{E}$ are of multiplicity one, $\omega$ must act as a scalar multiplication on them!

Case 1. Suppose $\operatorname{dim} V \geq 6$. Since $2 p=\operatorname{dim} \bigwedge^{2} V, \operatorname{dim} V \equiv 0,1$ (4). Hence, in fact, $\operatorname{dim} V \geq 8$ and $\operatorname{dim} \bar{\Lambda}^{2} V \geq 28$. So $p$ is much bigger than 6 which is quite critical, because for a proper choice of $m_{1} \geq m_{2} \geq m_{3} \geq \ldots$ there is a primitive $y_{m} \in \mathcal{E}$

$$
\begin{aligned}
y_{m}= & x_{12} \wedge x_{13} \wedge x_{14} \wedge \ldots \wedge x_{1 m_{1}} \wedge x_{23} \wedge x_{24} \\
& \wedge \ldots \wedge x_{2 m_{2}} \wedge x_{34} \wedge \ldots \wedge x_{q m_{q}} \in \wedge^{p} \wedge^{2} V
\end{aligned}
$$

which has $x_{34}$ as a factor. Let $S$ be the symmetric group on $g$-letters and let $(m, n)$ denote the cycle interchanging $m$ and $n$. Let $\Sigma \subset S$ be the union of the sets

$$
\begin{aligned}
& \left\{\left(m_{1}, m_{1}+i\right) \mid i=1, \cdots, g-m_{1}\right\}, \\
& \left\{\left(m_{2}, m_{2}+i\right) \mid i=1, \cdots, g-m_{2}\right\}, \\
& \{(3, *)(4, * *) \mid *<* *, 3<*, 4<* * \text { and }(*, * *) \text { does not appear as an index }
\end{aligned}
$$
in $\left.y_{m}^{\dot{i}}\right\}$.

For $\sigma \in \Sigma$ let $g_{\sigma} \in G L(V)$ be the unique element satisfying

$$
g_{\sigma}\left(x_{i}\right)=x_{\sigma(i)},
$$

and set $G_{\Sigma}=\left\{g_{\sigma} \mid \sigma \in \Sigma\right\} \subset G L(V)$.

Scaling $\omega$ if necessary, we can assume $\left.\omega\right|_{\left\langle y_{m}\right\rangle_{\bmod }}=$ Id. Since $\bigwedge^{p} \wedge^{2} G_{\Sigma}\left(y_{m}\right) \subset$ $\left\langle y_{m}\right\rangle_{\text {mod }}$ we thus have

$$
\omega\left(\bigwedge^{p} \Lambda^{2} g_{\sigma}\left(y_{m}\right)\right)=\Lambda^{p} \Lambda^{2} g_{\sigma}\left(y_{m}\right)
$$

Notice from (11) and the fact that $\omega$ acts as the identity on $\left\langle y_{m}\right\rangle_{\text {mod }}$ we have

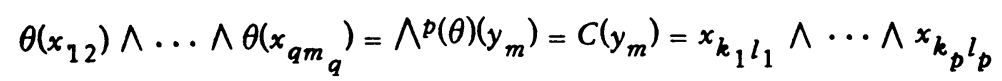

where the set $\left\{\left(k_{i}, l_{i}\right)\right\}_{i}$ complements the set of indices of $y_{m}$ in $\{(i, j) \mid 1 \leq i<$ $j \leq g\}$. Thus

$$
\theta\left(x_{12}\right) \in\left\langle x_{k_{1} l_{1}}, \cdots, x_{k_{p} l_{p}}\right\rangle .
$$

Observe from the construction of $\Sigma$ that $x_{12}$ is a factor of $\wedge^{p} \wedge^{2} g_{\sigma}\left(y_{m}\right)$ for all $\sigma \in \Sigma$. Thus

$$
\theta\left(x_{12}\right) \wedge \cdots=\Lambda^{p}(\theta)\left(\bigwedge^{p} \bigwedge^{2} g_{\sigma}\left(y_{m}\right)\right)=C\left(\bigwedge^{p} \wedge^{2} g_{\sigma}\left(y_{m}\right)\right)=x_{s_{1} t_{1}} \wedge \cdots \wedge x_{s_{p} t_{p}}
$$


where the pairs $\left(s_{i}, t_{i}\right), i=1, \cdots, p$, complement the pairs which appear in the standard decomposable vector $\bigwedge^{p} \bigwedge^{2} g_{\sigma}\left(y_{m}\right)$. As before, this gives

$$
\theta\left(x_{12}\right) \in\left\langle x_{s_{1} t_{1}}, \cdots, x_{s_{p} t_{p}}\right\rangle \text {. }
$$

Now intersecting the results of (15) as $\sigma$ runs over $\Sigma$ with the results of (14), we get $\theta\left(x_{12}\right)=0$ since equation (15) has the effect of eliminating one of the $x_{k_{i} l_{i}}$ of (14) from the basis of a subspace of $\left\langle x_{k_{1} l_{1}}, \cdots, x_{k_{p}} l_{p}\right\rangle$ which must contain $\theta\left(x_{12}\right)$. This contradiction completes Case 1 .

Case 2. Suppose $\operatorname{dim} V=5$. Here consider the primitive vector $y=x_{12} \wedge x_{13}$ $\wedge x_{14} \wedge x_{23} \wedge x_{24}$ and let $f_{2} \in \operatorname{sl}(V)$ be the element whose matrix with respect to $\left\{x_{i}\right\}$ has " 1 " in the $(3,2)$ position and zeros elsewhere. Then $f_{2}(y)=x_{12} \wedge$ $x_{13} \wedge x_{14} \wedge x_{23} \wedge x_{34} \in\langle y\rangle_{\text {mod }}$ and this vector can be used in place of the $y_{m}$ of Case 1 to complete the proof.

The remaining case to consider is $\operatorname{dim} V=4, \operatorname{dim} \bigwedge^{2} V=6, p=3$. Surprisingly it turns out here that there is a nontrivial automorphism $\rho \in$ Aut $G_{3}\left(\wedge^{2} V\right)$ which centralizes $G_{3}$, the image of $G L(V)$.

By way of preparation we first write $\wedge^{3} \wedge^{2} V$ as a sum of simple submodules. Keeping the same notation as in $\S 3$, let $\lambda_{1}, \lambda_{2}, \lambda_{3} \in H^{*}$ be the dual basis to $b_{1}$, $b_{2}, b_{3} \in H$. If $\lambda \in H^{*}$ is a dominant integral linear function (i.e., $\lambda\left(b_{i}\right)$ is a nonnegative integer [2, Chapter 7]) let $M_{\lambda}$ be the unique simple $s l(V)$-module of highest weight $\lambda$. If the $\Lambda_{i}$ 's are as in (8) then $\Lambda_{1}=\lambda_{1}, \Lambda_{2}=\lambda_{2}-\lambda_{1}, \Lambda_{3}=\lambda_{3}-$ $\lambda_{2}, \Lambda_{4}=-\Lambda_{1}-\Lambda_{2}-\Lambda_{3}$. Now $y_{1}=x_{12} \wedge x_{13} \wedge x_{14}$ and $y_{2}=x_{12} \wedge x_{13} \wedge x_{23}$ are primitive vectors and using the preceding identities we find that $w t\left(y_{1}\right)=$ $2 \lambda_{1}, w t\left(y_{2}\right)=2 \lambda_{3}$. Using Weyl's dimension formula [2, (40), (41), p. 257] it follows quickly that $\operatorname{dim} M_{2 \lambda_{1}}=10=\operatorname{dim} M_{2 \lambda_{3}}$. Also, since $\operatorname{dim} V=4, \operatorname{dim} \bigwedge^{3} \bigwedge^{2} V=20$, hence

$$
\bigwedge^{3} \wedge^{2} V=M_{1} \oplus M_{2} \text { where } M_{1}=\left\langle y_{1}\right\rangle_{\bmod }, M_{2}=\left\langle y_{2}\right\rangle_{\bmod }
$$

For the computations to follow we need bases of $M_{1}$ and $M_{2}$. Let $f_{i} \in \operatorname{sl}(V)$ be the element whose matrix in the basis $\left\{x_{i}\right\}$ has a " 1 " in the $(i+1, i)$ position and zeros elsewhere, and let $g_{\sigma}$ be as in (12). If $v \in \Lambda^{3} \Lambda^{2} V$ by $g_{\sigma}(v)$ we mean $\left(\wedge^{3} \wedge^{2} g_{\sigma}\right)(v)$ and by $f_{i}(v)$ we mean $d\left(\Lambda^{3} \circ \wedge^{2}\right)\left(f_{i}\right)(v)$. Then $M_{1}=\left\langle v_{i}\right\rangle_{i=1}^{10}$ and $M_{2}=\left\langle w_{i}\right\rangle_{i=1}^{10}$ where:

$$
\begin{aligned}
& v_{1}=x_{12} \wedge x_{13} \wedge x_{14}, \\
& v_{2}=-g_{(1,2)}\left(v_{1}\right)=x_{12} \wedge x_{23} \wedge x_{24}, \\
& v_{3}=-g_{(1,3)}\left(v_{1}\right)=x_{13} \wedge x_{23} \wedge x_{34}, \\
& v_{4}=-g_{(1,4)}\left(v_{1}\right)=x_{14} \wedge x_{24} \wedge x_{34},
\end{aligned}
$$




$$
\begin{aligned}
& v_{5}=f_{1}\left(v_{1}\right)=-x_{12} \wedge x_{14} \wedge x_{23}+x_{12} \wedge x_{13} \wedge x_{24}, \\
& v_{6}=f_{2}\left(v_{2}\right)=x_{13} \wedge x_{23} \wedge x_{24}+x_{12} \wedge x_{23} \wedge x_{34}, \\
& v_{7}=f_{3}\left(v_{3}\right)=x_{14} \wedge x_{23} \wedge x_{34}+x_{13} \wedge x_{24} \wedge x_{34} \\
& v_{8}=f_{2}\left(f_{1}\left(v_{1}\right)\right)=-x_{13} \wedge x_{14} \wedge x_{23}+x_{12} \wedge x_{13} \wedge x_{34}, \\
& v_{9}=f_{3}\left(f_{2}\left(v_{2}\right)\right)=x_{14} \wedge x_{23} \wedge x_{24}+x_{12} \wedge x_{24} \wedge x_{34}, \\
& v_{10}=f_{3}\left(f_{2}\left(f_{1}\left(v_{1}\right)\right)\right)=-x_{13} \wedge x_{14} \wedge x_{24}+x_{12} \wedge x_{14} \wedge x_{34}, \\
& w_{1}=x_{12} \wedge x_{13} \wedge x_{23}, \\
& w_{2}=g_{(1,4)}\left(w_{1}\right)=x_{23} \wedge x_{24} \wedge x_{34}, \\
& w_{3}=g_{(2,4)}\left(w_{1}\right)=x_{13} \wedge x_{14} \wedge x_{34}, \\
& w_{4}=g_{(3,4)}\left(w_{1}\right)=x_{12} \wedge x_{14} \wedge x_{24}, \\
& w_{5}=f_{3}\left(w_{1}\right)=x_{12} \wedge x_{14} \wedge x_{23}+x_{12} \wedge x_{13} \wedge x_{24}, \\
& w_{6}=f_{1}\left(f_{2}\left(f_{3}\left(w_{1}\right)\right)\right)=-x_{13} \wedge x_{23} \wedge x_{24}+x_{12} \wedge x_{23} \wedge x_{34}, \\
& w_{7}=f_{1}\left(w_{3}\right)=-x_{14} \wedge x_{23} \wedge x_{34}+x_{13} \wedge x_{24} \wedge x_{34}, \\
& w_{8}=f_{2}\left(f_{3}\left(w_{1}\right)\right)=x_{13} \wedge x_{14} \wedge x_{23}+x_{12} \wedge x_{13} \wedge x_{34}, \\
& w_{9}=f_{1}\left(f_{2}\left(w_{4}\right)\right)=-x_{14} \wedge x_{23} \wedge x_{24}+x_{12} \wedge x_{24} \wedge x_{34}, \text { and finally } \\
& w_{10}=f_{2}\left(w_{4}\right)=x_{13} \wedge x_{14} \wedge x_{24}+x_{12} \wedge x_{14} \wedge x_{34} \cdot
\end{aligned}
$$

We can now determine the centralizer of $G_{3}=\pi \Lambda^{3} \Lambda^{2} G L(V)$ in Aut $G_{3}\left(\wedge^{2} V\right)$ when $\operatorname{dim} V=4$. Let $\rho \in$ Aut $G_{3}\left(\wedge^{2} V\right)$ and suppose $\rho$ centralizes $G_{3}=\pi \wedge^{3} \wedge^{2} G L(V)$. Then $\rho=\pi(\omega)$ for some $\omega \in G L\left(\bigwedge^{3} \bigwedge^{2} V\right)$ and as in Lemma $6 \omega$ centralizes $G_{2}$. In other words, $\omega$ is an $s l(V)$-automorphism of $\Lambda^{3} \Lambda^{2} V$. Hence by (16), scaling $\omega$ if necessary, we can assume

$$
\left.\omega\right|_{M_{1}}=\mathrm{Id},\left.\quad \omega\right|_{M_{2}}=r \text { Id, } \quad r \in K:
$$

By Theorem 4 either $\omega=\bigwedge^{3}(\theta), \theta \in G L\left(\bigwedge^{2} V\right)$, or else $\omega=C \circ \bigwedge^{3}(\theta)$ where $C$ is given by (4). In the former case proceed as in the proof of Lemma 7 to get $\rho=$ Id. So suppose $\omega=C \circ \bigwedge^{3}(\theta)$, or equivalently, since $C^{2}=\mathrm{Id}, C \circ \omega=\Lambda^{3}(\theta)$.

Now $x_{23} \wedge x_{24} \wedge x_{34}=C \circ \omega\left(v_{1}\right)=\wedge^{3}(\theta)\left(v_{1}\right)=\theta\left(x_{12}\right) \wedge \theta\left(x_{13}\right) \wedge \theta\left(x_{14}\right)$, thus $\theta\left(x_{12}\right) \in\left\langle x_{23}, x_{24}, x_{34}\right\rangle$. Similarly, from the relation $C \circ \omega\left(v_{2}\right)=\bigwedge^{3}(\theta)\left(v_{2}\right)$ it follows that $\theta\left(x_{12}\right) \in\left\langle x_{13}, x_{14}, x_{34}\right\rangle$. Intersecting these two conditions on $\theta\left(x_{12}\right)$ we see that $\theta\left(x_{12}\right) \in\left\langle x_{34}\right\rangle$. In a similar monotonous fashion one can show

$$
\theta\left(x_{i j}\right) \in x_{k l} \text { whenever }\{i, j, k, l\}=\{1,2,3,4\} \text {. }
$$

Pick $\lambda_{i j} \in K^{\bullet}$ such that 


$$
\theta\left(x_{i j}\right)=\lambda_{i j} x_{k l} \text { where } i, j, k, l \text { are as in (18). }
$$

Using (19) and picking off coefficients from the identities $C \circ \omega\left(w_{1}\right)=\Lambda^{3}(\theta)\left(w_{1}\right)$, $C \circ \omega\left(v_{2}\right)=\Lambda^{3}(\theta)\left(v_{2}\right), C \circ \omega\left(w_{2}\right)=\Lambda^{3}(\theta)\left(w_{2}\right), C \circ \omega\left(v_{3}\right)=\Lambda^{3}(\theta)\left(v_{3}\right)$ we get the respective identities

$$
\begin{array}{ll}
-\tau=\lambda_{12} \lambda_{13} \lambda_{23}, & -1=\lambda_{12} \lambda_{23} \lambda_{24}, \\
-\tau=\lambda_{23} \lambda_{24} \lambda_{34}, & -1=\lambda_{13} \lambda_{23} \lambda_{34} .
\end{array}
$$

Dividing the third by the second, and the fourth by the first, we get the simultaneous relations

$$
\lambda_{34} / \lambda_{12}=\tau, \quad \lambda_{34} / \lambda_{12}=1 / \tau
$$

hence

$$
\tau^{2}=1, \quad \tau= \pm 1
$$

If $\tau=1, \omega=$ Id by $(17)$ and $\rho=\pi(\omega)=$ Id. Setting

$$
\begin{array}{lll}
\sigma\left(x_{12}\right)=-x_{34}, & \sigma\left(x_{13}\right)=-x_{24}, & \sigma\left(x_{14}\right)=-x_{23}, \\
\sigma\left(x_{23}\right)=x_{14}, & \sigma\left(x_{24}\right)=x_{13}, & \sigma\left(x_{34}\right)=x_{12},
\end{array}
$$

and letting

$$
\omega=C \circ \bigwedge^{3}(\sigma)
$$

one can check using the bases $\left\{v_{i}\right\}_{i},\left\{w_{i}\right\}_{i}$ of $M_{1}, M_{2}$ that

$$
\left.\omega\right|_{M_{1}}=I d,\left.\quad \omega\right|_{M_{2}}=- \text { Id }
$$

and $\omega$ centralizes $G_{2}$.

First Main Theorem. Let $\rho \in$ Aut $G_{p}\left(\Lambda^{2} V\right)$ where $G_{p}\left(\Lambda^{2} V\right)$ is the projective Grassmann variety of p-dimensional subspaces of $\Lambda^{2} V$ and $V$ is a finite-dimensional vector space over an algebraically closed field of characteristic zero. If $\rho$ centralizes the image of $G L(V)$ by $\pi \circ \bigwedge^{p} \bigwedge^{2}$ ( $\pi$ in Lemma 5 is the projection map), then $\rho=\mathrm{Id}$ except in the case $\operatorname{dim} V=4, p=3$ when $\rho=\operatorname{Id}$ or $\rho=\pi(\omega)$ where $\omega$ is given by (25), (24), (23) and (4).

Proof. Lemma 7, Lemma 13 and the discussion immediately preceding the statement of this theorem.

Second Main Theorem. There is only one algebraic duality theory.

Proof. By the First Main Theorem and the discussion at the end of $\$ 1$, there are only two possible algebraic duality theories: $D_{1}$ and $D_{2}$. Furthermore, they agree except possibly on the 7-dimensional metabelian algebras $N$ for which $\operatorname{cod} N^{2}=4$. By $[1, \S 7]$ there are only six isomorphism classes of such algebras. They correspond to the following elements of $\bigwedge^{3} \Lambda^{2} V$ : 


$$
\begin{array}{ll}
I_{1}=x_{12} \wedge x_{13} \wedge x_{23}, & I_{4}=x_{12} \wedge x_{34} \wedge\left(x_{14}-x_{23}\right), \\
I_{2}=x_{12} \wedge x_{13} \wedge x_{14}, & I_{5}=\left(x_{12}+x_{34}\right) \wedge x_{24} \wedge x_{14}, \\
I_{3}=x_{12} \wedge x_{13} \wedge x_{24}, & I_{6}=\left(x_{12}+x_{34}\right) \wedge x_{24} \wedge x_{13} .
\end{array}
$$

In terms of the bases $\left\{v_{i}\right\}_{i},\left\{w_{i}\right\}_{i}$ of $M_{1}, M_{2}$ (recall $\wedge^{3} \wedge^{2} V=M_{1} \oplus M_{2}$ ) we have $I_{1}=w_{1}, I_{2}=v_{1}, I_{3}=\left(v_{5}+w_{5}\right) / 2, I_{4}=\left(v_{6}+w_{6}\right) / 2-\left(v_{10}+w_{10}\right) / 2, I_{5}=$ $-\left(v_{4}+w_{4}\right), I_{6}=-\left(v_{5}+w_{5}\right) / 2-\left(v_{7}+w_{7}\right) / 2$. Hence if $\omega$ is as in the First Main Theorem, $\omega\left(I_{1}\right)=-w_{1}, \omega\left(I_{2}\right)=v_{1}, \omega\left(I_{3}\right)=\left(v_{5}-w_{5}\right) / 2, \omega\left(I_{4}\right)=\left(v_{6}-w_{6}\right) / 2-$ $\left(v_{10}-w_{10}\right) / 2, \omega\left(I_{5}\right)=\left(-v_{4}+w_{4}\right), \omega\left(I_{6}\right)=-\left(v_{5}-w_{5}\right) / 2-\left(v_{7}-w_{7}\right) / 2$.

Now to prove $D_{1}$ and $D_{2}$ are identical it is sufficient to find linear transformations $\sigma_{1}, \cdots, \sigma_{6}$ of $V$ satisfying $\langle\omega(I)\rangle=\left\langle\bigwedge^{3} \Lambda^{2}\left(\sigma_{j}\right)\left(I_{j}\right)\right\rangle$ for $j=1, \cdots, 6$. For $\sigma_{1}, \sigma_{2}$ it is clear we can pick the identity. For the rest we take:

$$
\begin{array}{lll}
\sigma_{3}\left(x_{i}\right)=x_{i}, i=1,2, & \sigma_{4}\left(x_{i}\right)=x_{i}, i=1,4, & \sigma_{5}\left(x_{i}\right)=x_{i}, i=3,4, \\
\sigma_{3}\left(x_{3}\right)=x_{4}, & \sigma_{4}\left(x_{2}\right)=x_{3}, & \sigma_{5}\left(x_{1}\right)=x_{2}, \\
\sigma_{3}\left(x_{4}\right)=x_{3}, & \sigma_{4}\left(x_{3}\right)=x_{2}, & \sigma_{5}\left(x_{2}\right)=x_{1},
\end{array}
$$

and $\sigma_{6}=\sigma_{3}$.

Corollary. The Scheuneman duality theory and the one given bere are identical.

Proof. It is straightforward to verify that Scheuneman's theory satisfies the axioms (I), .., (IV) of an algebraic duality theory. So the result follows from the preceding theorem.

\section{REFERENCES}

1. M. Gauger, On the classification of metabelian Lie algebras, Trans. Amer. Math. Soc. 179 (1973), 293-329.

2. N. Jacobson, Lie algebras, Interscience Tracts in Pure and Appl. Math., no. 10, Interscience, New York, 1962. MR 26 \#1345.

3. G. Leger and E. Luks, On a duality for metabelian Lie algebras, J. Algebra 21 (1972), 266-270.

4. J. Scheuneman, Two-step nilpotent Lie algebras, J. Algebra 7 (1967), 152-159. MR 36 \#225.

5. G. Seligman, Algebraic groups, Yale Lecture Notes, 1964.

6. J. Tits, Tabellen zu deneinfachen Lie Gruppen und ihren Darstellungen, Lecture Notes in Math., no. 40, Springer-Verlag, Berlin and New York, 1967. MR $36 \# 1575$.

7. R. Westwick, Linear transformations on Grassmann spaces, Pacific J. Math. 14 (1964), 1123-1127. MR $29 \# 4766$.

DEPARTMENT OF MATHEMATICS, UNIVERSITY OF MASSACHUSETTS, AMHERST, MASSACHUSETTS 01002 